\title{
Improved HCN channels in pyramidal neurons and their new expression levels in pericytes and astrocytes in the gerbil hippocampal CA1 subfield following transient ischemia
}

\author{
JOON HA PARK ${ }^{1 *}$, DAE WON KIM ${ }^{2 *}$, TAE-KYEONG LEE ${ }^{3}$, CHEOL WOO PARK $^{3}$, YOUNG EUN PARK $^{3}$, \\ JI HYEON AHN ${ }^{4}$, HYANG-AH LEE ${ }^{5}$, MOO-HO WON ${ }^{3 *}$ and CHOONG-HYUN LEE ${ }^{6 *}$
}

\begin{abstract}
${ }^{1}$ Department of Anatomy, College of Korean Medicine, Dongguk University, Gyeongju, Gyeongbuk 38066;
${ }^{2}$ Department of Biochemistry and Molecular Biology, Research Institute of Oral Sciences, College of Dentistry, Gangneung-Wonju National University, Gangneung, Gangwon 25457; ${ }^{3}$ Department of Neurobiology, School of Medicine, Kangwon National University, Chuncheon, Gangwon 24341; ${ }^{4}$ Department of Biomedical Science, Research Institute of Bioscience and Biotechnology, Hallym University, Chuncheon, Gangwon 24252; 5 Department of Obstetrics and Gynecology, School of Medicine, Kangwon National University, Chuncheon, Gangwon 24341; ${ }^{6}$ Department of Pharmacy, College of Pharmacy, Dankook University, Cheonan, Chungcheongnam 31116, Republic of Korea
\end{abstract}

Received May 8, 2019; Accepted September 4, 2019

DOI: $10.3892 /$ ijmm.2019.4353

\begin{abstract}
Hyperpolarization-activated cyclic nucleotide-gated $(\mathrm{HCN})$ channels have been known to participate in the regulation of neuronal excitability, synaptic transmission and long-term potentiation in the hippocampus. The present study investigated transient ischemia-induced changes of $\mathrm{HCN} 1$ and HCN2 expressions in the Cornu Ammonis 1 (CA1) subfield of the hippocampus in gerbils subjected to 5 min transient global cerebral ischemia $(\operatorname{tgCI})$. Neuronal death was exhibited in pyramidal neurons of the striatum pyramidale in the CA1 subfield 4 days after tgCI. HCN1 and HCN2 immunoreactivities were demonstrated in intact CA1 pyramidal neurons, and were transiently and markedly increased in the CA pyramidal neurons at $6 \mathrm{~h}$ after ischemia. Thereafter, they gradually decreased in a time-dependent manner. A total of 4 days after ischemia, $\mathrm{HCN} 1$ and $\mathrm{HCN} 2$ immunoreactivities were barely
\end{abstract}

Correspondence to: Professor Moo-Ho Won, Department of Neurobiology, School of Medicine, Kangwon National University, 1 Gangwondaehak Road, Chuncheon, Gangwon 24341, Republic of Korea

E-mail: mhwon@kangwon.ac.kr

Professor Choong-Hyun Lee, Department of Pharmacy, College of Pharmacy, Dankook University, 119 Dandae Road, Cheonan, Chungcheongnam 31116, Republic of Korea

E-mail: anaphy@dankook.ac.kr

*Contributed equally

Key words: transient global cerebral ischemia, hyperpolarizationactivated cyclic nucleotide-gated channel, astrocyte, pericyte, hippocampus, delayed neuronal death detected in the CA1 pyramidal neurons; however, HCN1 and $\mathrm{HCN} 2$ were began to be expressed in pericytes and astrocytes at 4 days after ischemia. The results indicated that $\mathrm{HCN} 1$ and $\mathrm{HCN} 2$ expression levels were apparently changed in the gerbil hippocampal CA1 subfield following tgCI and suggested that ischemia-induced alterations in $\mathrm{HCN} 1$ and $\mathrm{HCN} 2$ expression levels may be closely associated with the death of CA1 pyramidal neurons following $5 \mathrm{~min}$ of $\operatorname{tgCI}$.

\section{Introduction}

A brief period of global brain ischemia causes neuronal death/loss in vulnerable regions a few days following reperfusion. Pyramidal neurons in the Cornu Ammonis 1 (CA1) subfield of the hippocampus proper are known to be the most significantly affected neurons following $5 \mathrm{~min}$ of transient global cerebral ischemia (tgCI); $\operatorname{tgCI}$-induced death of pyramidal neurons in the CA1 subfield occurs at 4-5 days following $\operatorname{tgCI}$, therefore this characteristic phenomenon is termed as delayed neuronal death (DND) (1). Numerous factors participate in the process of the tgCI-induced DND. For example, glutamate-mediated excitotoxicity, oxidative stress and neuroinflammatory process including activation of glial cells are involved in DND (2-6).

Hyperpolarization-activated cation currents $\left(I_{\mathrm{h}}\right)$, which are conducted by hyperpolarization-activated cyclic nucleotide-gated (HCN) channels, serve roles in the regulation of neuronal properties including neuronal rhythmic activity (7). In the hippocampus, $I_{\mathrm{h}}$ and $\mathrm{HCN}$ channels have been established to participate in controlling neuronal excitability, dendritic integration, synaptic transmission and plasticity, long-term potentiation, and learning and memory formation (8-13). Until now, 4 subunits of $\mathrm{HCN}$ channels (HCN1, HCN2, HCN3 and $\mathrm{HCN} 4$ ) have been identified, and they are expressed in mammalian brains (14). Among them, $\mathrm{HCN} 1$ conducts $I_{\mathrm{h}}$ with 
fast kinetics and modest cAMP gating, whereas HCN2, which is strictly regulated by cAMP, conducts slow-kinetic $I_{\mathrm{h}}(15-17)$.

It has been demonstrated that alterations in functions and/or expressions of HCN channels are associated with abnormal and pathological hyperexcitability $(18,19)$. A previous study has suggested that the expression levels of HCN channels are increased in reactive astrocytes in the rat brain following a permanent occlusion of the middle cerebral artery, which causes permeant focal cerebral ischemia (20). In the present study, time-dependent changes of $\mathrm{HCN} 1$ and $\mathrm{HCN} 2$ protein expression levels in the hippocampal CA1 subfield, which is the most vulnerable to tgCI, were investigated following $5 \mathrm{~min}$ of $\operatorname{tgCI}$ in gerbils.

\section{Materials and methods}

Experimental animals. The 6-month old male Mongolian gerbils (Meriones unguiculatus) were obtained from the Experimental Animal Center, Kangwon National University. All experimental procedures were approved by the Institutional Animal Care and Use Committee at Kangwon National University (approval no. KW-180124-1). The numbers of animals used in this study and the suffering caused by the procedures used in this experiment were minimized

Induction of $\operatorname{tgCI}$. As described in the method of our previous studies $(2,21,22)$, the surgical procedure for $\operatorname{tgCI}$ was performed as follows: Briefly, gerbils (total $n=84$ ) were anesthetized with a mixture of $2.5 \%$ isoflurane in oxygen $(34 \%)$ and nitrous oxide $(66 \%)$, with a modification of methods of previous studies (23-26), and level of anesthesia was confirmed by pedal reflex (firm toe pinch). Blood flow to the brain was completely interrupted through the occlusion of bilateral common carotid arteries for $5 \mathrm{~min}$, and confirmed by the observation of the inhibition of blood flow in the central artery in retinae using an ophthalmoscope (HEINE Optotechnik). The body temperature was monitored with a rectal temperature probe and controlled under normothermic $\left(37 \pm 0.5^{\circ} \mathrm{C}\right)$ conditions using a thermometric blanket prior and subsequent to surgery. Sham gerbils were subjected to the same procedure without the bilateral common carotid artery occlusion. No tgCI-induced mortality occurred in any of the sham- or ischemia-operated groups.

Western blot analysis. $\mathrm{HCN} 1$ and $\mathrm{HCN} 2$ protein levels were analyzed in the CA1 subfield at designated times (sham, 6, 12 h, 1, 2 and 4 days after tgCI) using western blot analysis. The designated time periods (from $6 \mathrm{~h}$ to 4 days after $\operatorname{tgCI}$ ) were selected as ischemia-induced neuronal death of CA1 pyramidal neurons occurred $\sim 4$ days after tgCI and because significant changes in various factors, which were important in neuronal survival and death, were significantly altered prior to the occurrence of ischemia-induced neuronal death $(21,22)$. As described in our previously published method (22), 7 animals at each time point were anaesthetized with intraperitoneal injection of sodium pentobarbital $(60 \mathrm{mg} / \mathrm{kg})$ (JW Pharmaceutical Co., Ltd.) (25-28), and their brains were removed. The removed brains were serially and transversely cut into $400 \mu \mathrm{m}$ thickness on a vibratome (Leica Microsystems $\mathrm{GmbH}$ ). Tissues of the CA1 subfield were dissected from the hippocampi with a surgical blade and homogenized in $50 \mathrm{mM}$ PBS
(pH 7.4) containing 0.1 mM EGTA ( $\mathrm{pH} 8.0$ ), 0.2\% Nonidet P-40, 10 mM EDTA ( $\mathrm{pH} 8.0$ ), $15 \mathrm{mM}$ sodium pyrophosphate, $100 \mathrm{mM} \beta$-glycerophosphate, $50 \mathrm{mM} \mathrm{NaF}, 150 \mathrm{mM} \mathrm{NaCl}$, $2 \mathrm{mM}$ sodium orthovanadate, $1 \mathrm{mM}$ phenylmethylsulfonyl fluoride and $1 \mathrm{mM}$ dithiothreitol (DTT). HCN1 and HCN2 protein concentrations were determined using a Micro BCA protein assay kit (Pierce; Thermo Fisher Scientific, Inc.) after the homogenized tissues were centrifugated at $16,000 \mathrm{xg}$ for $20 \mathrm{~min}$ at $4^{\circ} \mathrm{C}$. Aliquot containing total protein $(20 \mathrm{mg})$ was boiled in loading buffer containing $150 \mathrm{mM}$ Tris-HCI (pH 6.8), 6\% SDS, 3 mM DTT, $0.3 \%$ bromophenol blue and $30 \%$ glycerol and then loaded onto $10 \%$ polyacrylamide gel. The gel was transferred to nitrocellulose transfer membranes (Pall Corporation) following electrophoresis. To reduce background staining, the membranes were blocked with 5\% non-fat dry milk in PBS containing 0.1\% Tween-20 (PBST) for $45 \mathrm{~min}$ at room temperature. After washing three times with PBST (each for $5 \mathrm{~min}$ ), the membrane was incubated with rabbit anti-HCN1 (1:400; cat. no. MABN20; EMD Millipore), rabbit anti-HCN2 (1:400; cat. no. MAB5596; EMD Millipore), and mouse anti- $\beta$-actin (1:5,000; cat. no. A5316; Sigma-Aldrich; Merck KGaA) antibodies overnight at $4^{\circ} \mathrm{C}$. Finally, the membrane was exposed to horseradish peroxidase-conjugated goat anti-rabbit secondary antibody (1:5,000; cat. no. 12-348; Sigma-Aldrich; Merck KGaA) or horseradish peroxidase-conjugated goat anti-mouse secondary antibody (1:4,000; cat. no. sc-2031; Santa Cruz Biotechnology, Inc.) for $2 \mathrm{~h}$ at room temperature and visualized with an enhanced chemiluminescence kit (GE Healthcare Life Sciences). Results of the western blot analysis were scanned, and densitometric quantification of the bands was performed using Image J 1.46 software (National Institutes of Health). The quantification was represented by relative optical density (ROD). A ratio of the ROD was calibrated as percentages: The sham group was designated as $100 \%$.

Tissue processing for histology. Brain sections containing the hippocampus of each group $(n=7$ for each point in time) were prepared at designated times (sham, 6, $12 \mathrm{~h}, 1,2$ and 4 days after $\operatorname{tgCI}$ ). According to the method described in our previous studies $(2,21,22)$, the brain tissues were fixed by the perfusion of $4 \%$ paraformaldehyde (in $0.1 \mathrm{M} \mathrm{PBS}$ ) through the ascending aorta. Then, the brains were removed and post-fixed with the same fixative for $8 \mathrm{~h}$ at room temperature, then cryoprotected by infiltration with $30 \%$ sucrose. Finally, the brain tissues were serially sectioned into $25-\mu \mathrm{m}$ thickness of coronal sections in a cryostat (Leica Microsystems $\mathrm{GmbH}$ ) for subsequent analysis.

Cresyl violet $(C V)$ staining. To examine change in the distribution pattern and morphology of cells or neurons in the hippocampus following tgCI, the fixed sections were stained with $\mathrm{CV}$, as we descried previously (29). In brief, we made a solution of $1.0 \%$ (w/v) CV acetate (Sigma) and added glacial acetic acid (Sigma) to the solution. The sections were stained with the CV solution for $2 \mathrm{~min}$, washed and dehydrated by immersing in ethanol.

Immunohistochemistry. According to the methods described in our previous studies $(2,21,22)$, immunohistochemical 


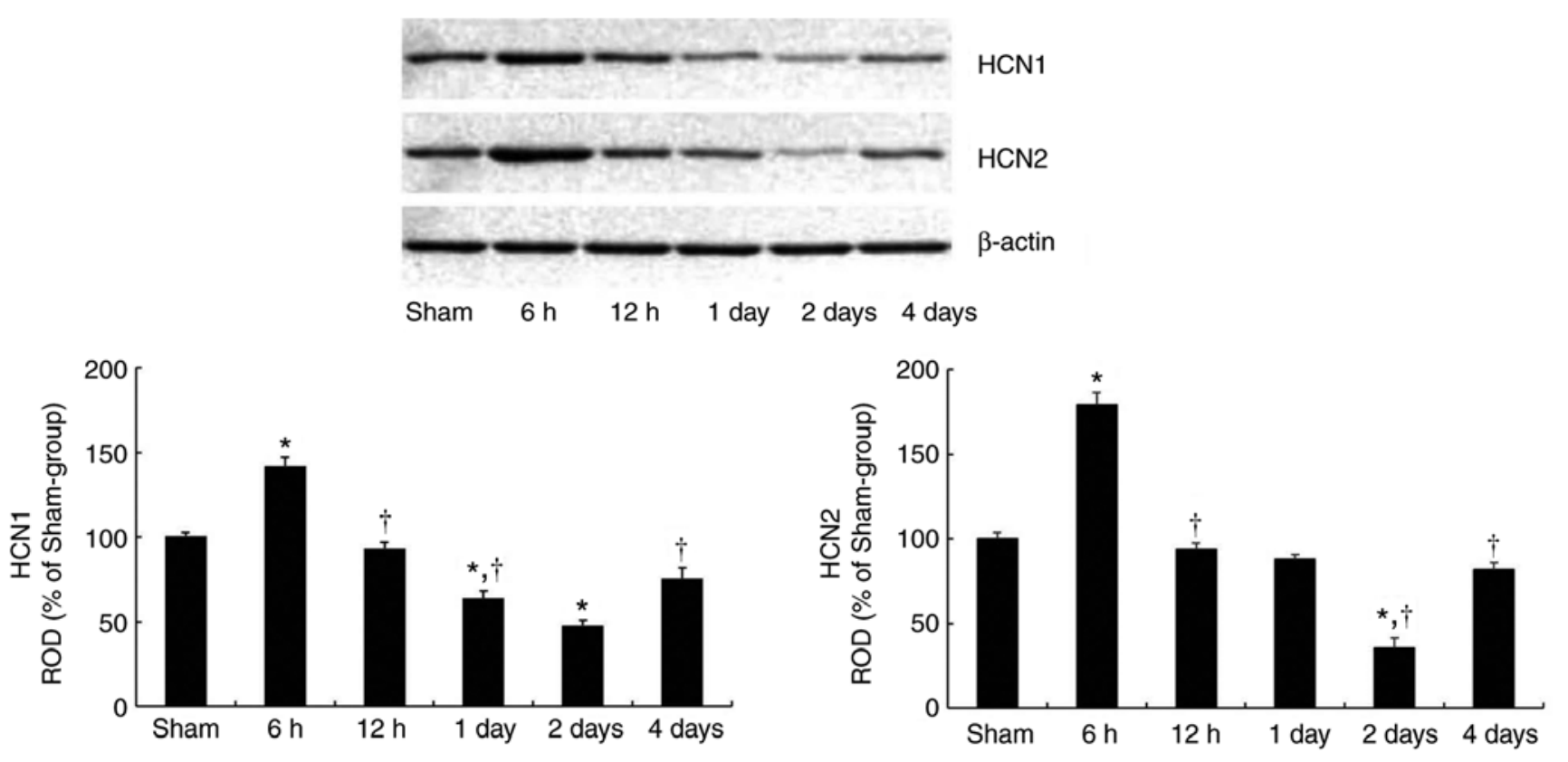

Figure 1. Western blot analysis of HCN1 and HCN2 in the CA1 subfield of the hippocampus in the sham-operated and ischemia-operated groups. ROD is presented as a percentage value of immunoblot band ( $\mathrm{n}=7$ at each time point). ${ }^{*} \mathrm{P}<0.05$ vs. the sham-operated-group. ${ }^{\dagger} \mathrm{P}<0.05$ vs. the previous timepoint group. Bars indicate the mean \pm standard error of the mean. HCN, hyperpolarization-activated cyclic nucleotide-gated channel; CA1, Cornu Ammonis 1 .

staining for HCN1 and HCN2 was performed. Briefly, the fixed sections were incubated with rabbit anti-HCN1 (1:100; EMD Millipore) or rabbit anti-HCN2 (1:100; EMD Millipore) as primary antibodies. The sections were then exposed to biotinylated goat anti-rabbit IgG (1:200; Vector Laboratories, Inc.) and streptavidin peroxidase complex (1:200; Vector Laboratories, Inc.). Finally, the sections were visualized with 3,3'-diaminobenzidine (in $0.1 \mathrm{M}$ Tris- $\mathrm{HCl}$ buffer). In order to confirm the specificity of each immunoreaction, a negative control test was performed using pre-immune serum instead of each primary antibody. No immunoreactivity was observed in the negative control tests.

For the quantitative analysis of HCN1 and HCN2 immunoreactivity, 6 sections with $120 \mu \mathrm{m}$ intervals per animal were selected. According to our published method (22), digital images of the HCN1 and HCN2 immunoreactive structures were captured in the hippocampus with Axio Imager 2 microscope (Carl Zeiss AG) equipped with an Axiocam digital camera (Carl Zeiss AG). The captured images were calibrated into an array of $512 \times 512$ pixels. The density of HCN1- and HCN2-immunoreactive structures was evaluated as an optical density (OD). Each OD was obtained following the transformation of the mean gray level using the formula: $O D=\log$ (256/mean gray level). The background was captured from areas adjacent to the measured area and subtracted. Finally, a ratio of the OD of image file was calibrated as \% (relative optical density, ROD) and analyzed using NIH Image 1.59 software (National Institutes of Health). A ratio of the ROD was calibrated as percentages, with the sham group representing $100 \%$.

Double immunofluorescence staining. To examine the cell type (neurons or glial cells) exhibiting HCN1 or HCN2 immunoreactivity following tgCI, double immunofluorescence staining was performed according to our previously published protocol $(21,22)$. In brief, rabbit anti-HCN1 (1:50; cat. no. MABN20; EMD Millipore) or rabbit anti-HCN2 (1:50, cat. no. MAB5596; Millipore)/mouse anti-ATP-sensitive inward rectifier potassium channel 8 (Kir6.1; 1:100; cat. no. NBP2-59324; Novus Biologicals, LLC) antibodies were selected to identify pericytes or mouse anti-glial fibrillary acidic protein (GFAP; 1:200; cat. no. AB5804; EMD Millipore) antibodies were used to identify astrocytes. The fixed sections were then incubated in the mixture of antisera and reacted in a mixture containing fluorescein isothiocyanate-conjugated goat anti-rabbit IgG (1:200; cat. no. 111-095-003; Jackson ImmunoResearch, West Grove, PA, USA) and Cy3-conjugated goat anti-mouse IgG (1:200; cat. no. 115-165-003; Jackson ImmunoResearch Laboratories Inc.). Finally, the immunoreactions were observed by using a LSM510 META NLO confocal microscope (Carl Zeiss AG) at x20 magnification.

Statistical analysis. The data are presented as the means \pm standard error of the means. Differences of the means among the groups were analyzed by analysis of variance followed by Tukey's Honest Significance Difference test, using SPSS 17.0 software (IBM Corp.). $\mathrm{P}<0.05$ was considered to indicate a statistically significant difference.

\section{Results}

tgCI-induced changes in HCN1 and HCN2 protein levels. $\mathrm{HCN} 1$ and HCN2 protein levels in the hippocampal CA1 subfield became significantly altered with time following tgCI (Fig. 1). HCN1 ( $\mathrm{P}=0.0182)$ and $\mathrm{HCN} 2(\mathrm{P}=0.0076)$ protein levels were significantly increased at $6 \mathrm{~h}$ after tgCI compared with those in the sham-operated group. Thereafter, the protein levels of HCN1 and HCN2 gradually decreased in a time-dependent manner until 2 days after tgCI. And then, at 4 days after $\operatorname{tgCI}$, the HCN1 $(\mathrm{P}=0.0324)$ and $\mathrm{HCN} 2(\mathrm{P}=0.0194)$ 

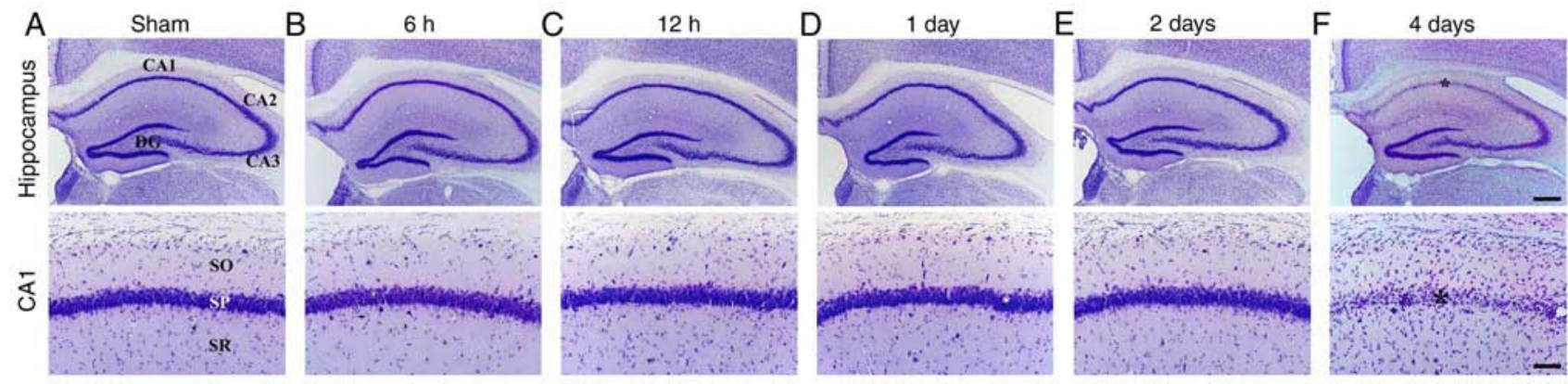

Figure 2. CV staining of the hippocampus of the (A) sham and (B-F) ischemia-operated groups. High magnification images of the CA1 regions are shown in the lower panels. A significant loss of $\mathrm{CV}^{+}$cells was demonstrated in the SP of the CA1 subfield at 4 days after tgCI. Scale bar, $400 \mu \mathrm{m}$ (upper panels) and $50 \mu \mathrm{m}$ (lower panels). Asterisks represent the $\mathrm{CV}^{+}$cells. CV, cresyl violet; CA, Cornu Ammonis; DG, dentate gyrus; SO, stratum oriens; SP, stratum pyramidale; $\mathrm{SR}$, stratum radiatum.

protein levels were significantly increased compared with those at 2 days after $\operatorname{tgCI}$.

tgCI-induced delayed neuronal death. Ischemia-induced DND in the hippocampus was observed in the CA1 subfield at 4 days after tgCI using CV staining (Fig. 2). In the sham-operated group, $\mathrm{CV}$ staining demonstrated the normal distribution of hippocampal cells in all hippocampal subregions (Fig. 2A). In the ischemia-operated group, the distribution pattern of $\mathrm{CV}$-positive $\left(\mathrm{CV}^{+}\right)$cells was not altered in the hippocampus until 2 days after tgCI (Fig. 2B-E). However, a significant loss of $\mathrm{CV}^{+}$cells was observed in the stratum pyramidale of the CA1 subfield, not the other subregions, at 4 days after tgCI (Fig. 2F).

\section{tgCI-induced change in HCN1 immunoreactivity}

CA1 subfield. In the sham-operated group, HCN1 immunoreactivity was primarily detected in neurons of the stratum pyramidale in the CA1 subfield, which are called CA1 pyramidal cells or neurons (Fig. 3A). In the ischemia-operated group, HCN1 immunoreactivity in the CA1 pyramidal neurons was markedly increased $(\mathrm{P}=0.0022)$ at $6 \mathrm{~h}$ after tgCI (Fig. 3B and G). Thereafter, HCN1 immunoreactivity in the CA1 pyramidal neurons was gradually decreased in a time-dependent manner (Fig. 3C-G). HCN1 immunoreactivity in the CA1 pyramidal neurons was barely detected in the CA1 pyramidal neurons at 4 days after tgCI; however, at this time interval, HCN1 immunoreactivity began to be exhibited in cells in the striata oriens and radiatum, which are non-pyramidal cells (Fig. 3F). Their cell type were examined by double immunofluorescence staining and it was identified that HCN1 immunoreactivity was colocalized with Kir6.1-immunoreactive cells (pericytes) and GFAP-immunoreactive cells (astrocytes), which are components of the blood brain barrier (BBB), in the striatum radiatum at 4 days after $\operatorname{tgCI}$ (Fig. $3 \mathrm{H}-\mathrm{M}$ ).

CA3 subfield. In the sham-operated group, HCN1 immunoreactivity was clearly demonstrated in pyramidal neurons of the hippocampal CA3 subfield (Fig. 4A). In the ischemia-operated group, the level of HCN1 immunoreactivity was not significantly altered in CA3 pyramidal neurons after tgCI (Fig. 4B-G).

\section{tgCI-induced changes in HCN2 immunoreactivity}

CAl subfield. In the sham-operated group, HCN2 immunoreactivity was identified in the pyramidal neurons and non-pyramidal cells of the hippocampal CA1 subfield (Fig. 5A). In the ischemia-operated group, HCN2 immunoreactivity in pyramidal neurons and non-pyramidal cells of the CA1 subfield was significantly $(\mathrm{P}<0.001)$ and transiently increased at $6 \mathrm{~h}$ after tgCI and then gradually decreased until 2 days after $\operatorname{tgCI}$ (Fig. 5B-E and G). At 4 days after $\operatorname{tg} \mathrm{CI}$, $\mathrm{HCN} 2$ immunoreactivity was significantly decreased in the CA1 pyramidal neurons, indicating that HCN2 immunoreactivity was exhibited in the nuclei of certain CA1 pyramidal neurons and many non-pyramidal cells (Fig. 5F). At this time interval, the type of non-pyramidal cells was examined by double immunofluorescence staining and it was identified that HCN2 immunoreactivity was colocalized in the Kir6.1-immunoreactive pericytes and GFAP-immunoreactive astrocytes (Fig. 5H-M).

CA3 subfield. In the sham-operated group, HCN2 immunoreactivity was clearly observed in the pyramidal neurons of the hippocampal CA3 subfield (Fig. 6A). In the ischemia-operated group, no significant change in HCN2 immunoreactivity was observed in the CA3 pyramidal neurons after tgCI (Fig. 6B-G).

\section{Discussion}

In our recently published study, it was demonstrated that unilateral common carotid artery occlusion in gerbils produced infarcts or selective neuronal death in a number of areas of the brain, in which the pattern of neuronal death was significantly different from that in transient global cerebral ischemia induced by bilateral common carotid artery occlusion (30). Therefore, in the present study, tgCI was induced by bilateral common carotid artery occlusion in gerbils. In addition, all gerbils of the experimental groups survived. This result was in accordance with a previous study that demonstrated that the survival rate of gerbils following $5 \mathrm{~min}$ of $\operatorname{tgCI}$ was $100 \%$ (31). Furthermore, in the present study, ischemia-induced DND in the hippocampus was observed in the CA1 subfield at 4 days after tgCI, which was consistent with our previous studies $(21,22)$.

HCN channels have been established to be predominantly localized in neurons, not in glial cells, in the mouse cortex and rat hippocampus, and $I h$ in hippocampal CA1 neurons is primarily mediated by $\mathrm{HCN} 1$ and $\mathrm{HCN} 2(16,17,20,32,33)$. In addition, it has been suggested that HCN1 and HCN2 mRNAs are expressed in interneurons and in principal neurons of the 

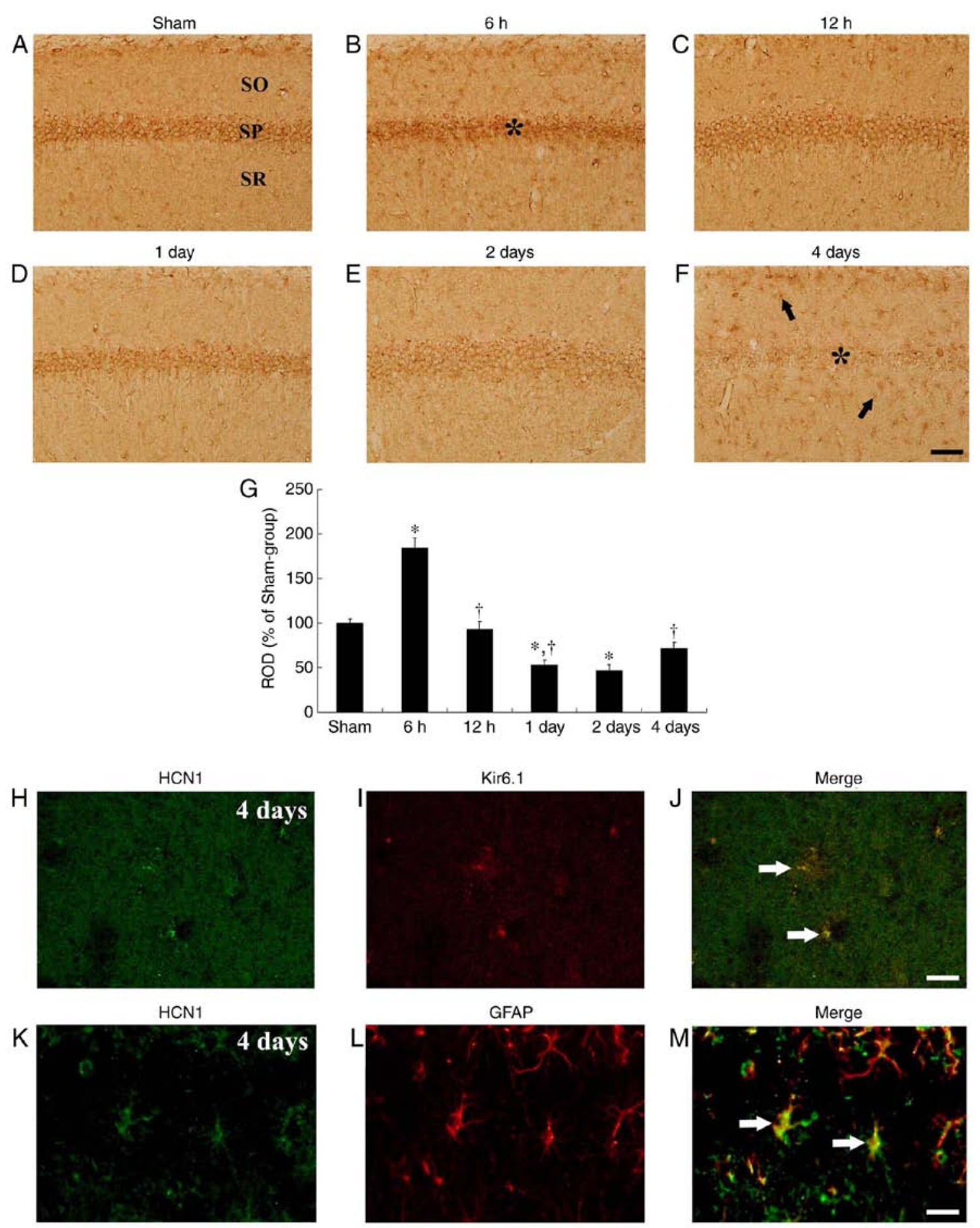

Figure 3. HCN1 immunohistochemistry in the hippocampal CA1 region. (A-F) HCN1 immunohistochemistry in the CA1 subfield of the (A) sham and (B-F) ischemia-operated groups at (B) $6 \mathrm{~h},(\mathrm{C}) 12 \mathrm{~h}$, (D) 1 day, (E) 2 days and (F) 4 days. HCN1 immunoreactivity was markedly increased in the CA1 pyramidal neurons $6 \mathrm{~h}$ after tgCI. Thereafter, $\mathrm{HCN} 1$ immunoreactivity is decreased in a time-dependent manner and was barely observed in the CA1 pyramidal neurons 4 days after tgCI. Scale bar, $50 \mu \mathrm{m}$. The asterisks and the arrows represent CA1 pyramidal neurons and non-pyramidal cells, respectively. (G) ROD is presented as percentages of $\mathrm{HCN} 1$ immunoreactive structures in the $\mathrm{CA} 1$ subfield following $\operatorname{tgCI}\left(\mathrm{n}=7\right.$ at each point in time. ${ }^{*} \mathrm{P}<0.05$ vs. the sham-operated group, ${ }^{\dagger} \mathrm{P}<0.05$ vs. the previous timepoint group. Bars indicate the means \pm standard error of the mean. (H-M) Double immunofluorescence staining for $(\mathrm{H}$ and $\mathrm{K}) \mathrm{HCN} 1$ (green), (I) Kir6.1 (red), (L) GFAP (red) and (J and M) merged images in the stratum pyramidale 4 days after tgCI. HCN1 immunoreactivity was localized in Kir6.1-immunoreactive pericytes and GFAP-immunoreactive astrocytes. Arrows denote Kir6.1-immunoreactive pericytes and GFAP-immunoreactive astrocytes in the respective images. Scale bar, $20 \mu \mathrm{m}$. HCN1, hyperpolarization-activated cyclic nucleotide-gated 1; CA1, Cornu Ammonis 1; tgCI, transient global cerebral ischemia; ROD, relative optical density; Kir6.1, ATP-sensitive inward rectifier potassium channel 8; GFAP, anti-glial fibrillary acidic protein.

hippocampus in rodents $(16,32)$. In particular, Notomi and Shiqemoto (34) revealed that the pyramidal cell layer of all hippocampal subfields in rats exhibited moderate to intense HCN1 and HCN2 immunoreactivity levels. These results are consistent with the results of the present study, which indicated that $\mathrm{HCN} 1$ and $\mathrm{HCN} 2$ immunoreactivities were observed in pyramidal neurons and interneurons, not in glial cells, of the hippocampal CA1 subfield in the sham-operated-group. 

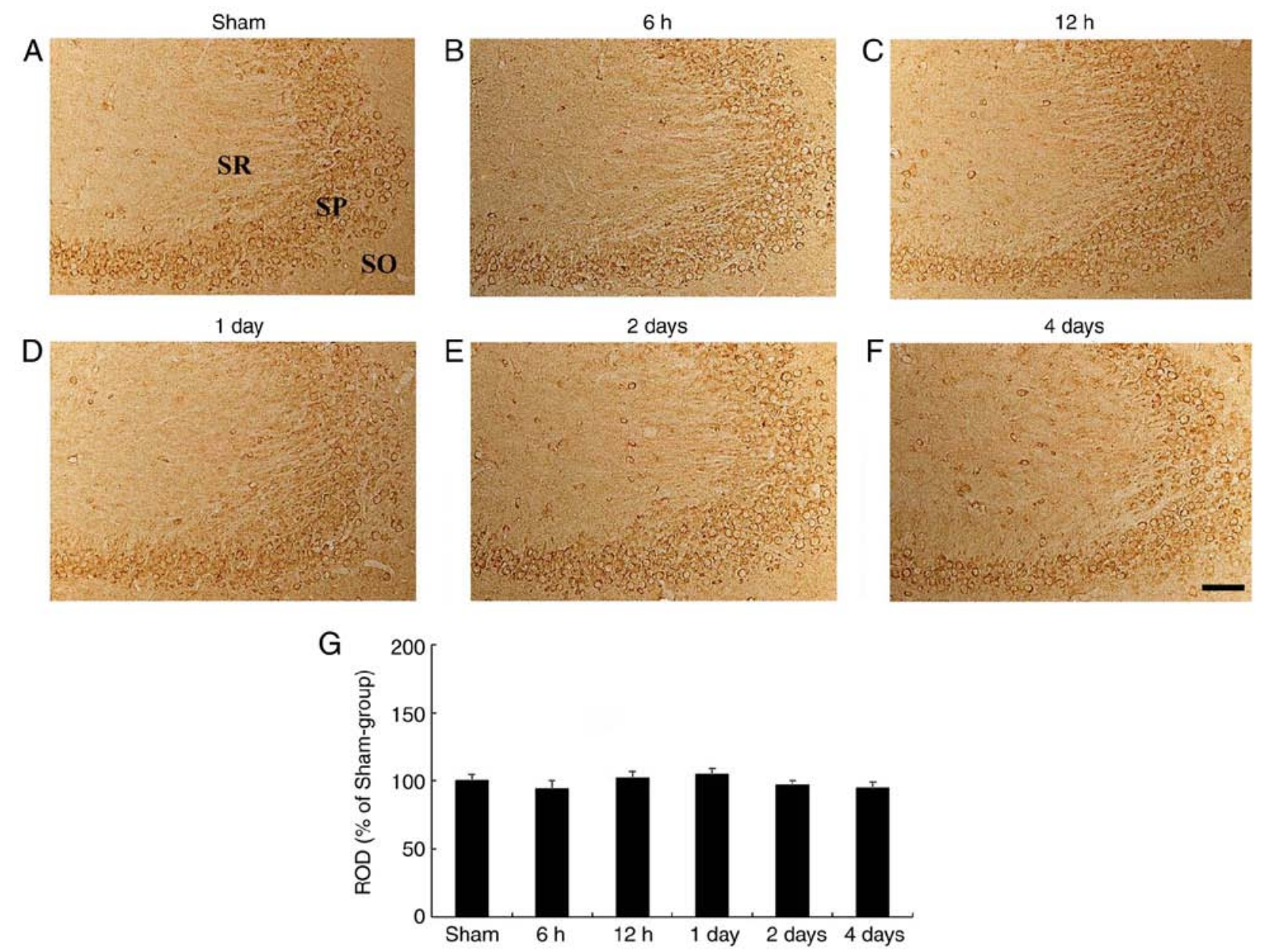

Figure 4. HCN1 immunohistochemistry in the hippocampal CA3 region. (A-F) HCN1 immunohistochemistry in the CA3 subfield of the (A) sham- and ischemia-operated (B-F) groups at (B) $6 \mathrm{~h}$, (C) $12 \mathrm{~h}$, (D) 1 day, (E) 2 days and (F) 4 days. HCN1 immunoreactivity in the CA3 subfield was not significantly changed in any of the experimental groups. Scale bar, $50 \mu \mathrm{m}$. (G) ROD is presented as percentages of HCN1 immunoreactive structures in the CA3 subfield following $\operatorname{tgCI}(\mathrm{n}=7$ at each point in time). $\mathrm{HCN} 1$, hyperpolarization-activated cyclic nucleotide-gated 1; CA3, Cornu Ammonis 3; SO, stratum oriens; $\mathrm{SP}$, stratum pyramidale; SR, stratum radiatum; ROD, relative optical density; tgCI, transient global cerebral ischemia.

It has been demonstrated that the expression levels of $\mathrm{HCN}$ genes are significantly increased in mouse brain tissue 2 weeks after focal cerebral ischemia (20). Conversely, another study suggested that HCN1 expression was significantly decreased in the hippocampal CA1 subfield and neocortex of the rat following chronic cerebral hypoperfusion; this study concluded that downregulated $\mathrm{HCN} 1$ expression may lead to impairment of learning and memory function (35). In animal models of epilepsy, Oh et al (36) indicated that HCN1, not HCN2, immunoreactivity was transiently and significantly increased in the rat hippocampus at $30 \mathrm{~min}$ and $12 \mathrm{~h}$ following pilocarpine-induced status epilepticus and suggested that enhanced HCN1 immunoreactivity may result from elevated hippocampal excitability for compensatory responses, as hippocampal circuit activity may be important for a regulation of $\mathrm{HCN}$ expression $(36,37)$. In the present study, it was identified that $\mathrm{HCN} 1$ and $\mathrm{HCN} 2$ immunoreactivity in the CA1 pyramidal neurons were transiently and markedly increased at $6 \mathrm{~h}$ after tgCI and decreased in a time-dependent manner thereafter. Therefore, it may be hypothesized that transient elevations of $\mathrm{HCN} 1$ and $\mathrm{HCN} 2$ immunoreactivities in CA1 pyramidal neurons at $6 \mathrm{~h}$ after $\operatorname{tg} \mathrm{CI}$ may be one of compensatory responses to the tgCI-induced alteration in hippocampal excitability and that time-dependent decreases of HCN1 and $\mathrm{HCN} 2$ immunoreactivities thereafter in the CA1 pyramidal neurons may be associated with the process of tgCI-induced DND.

In the present study, it was identified that HCN1 and HCN2 immunoreactivities in the CA1 pyramidal neurons were barely detected at 4 days after tgCI; however, at this time point, HCN1 and HCN2 immunoreactivities were observed in Kir6.1-immunoreactive pericytes and GFAP-immunoreactive astrocytes. Unfortunately, why HCN1 and HCN2 immunoreactivity began to be expressed in pericytes and astrocytes in the ischemic CA1 subfield 4 days after tgCI cannot be explained clearly. However, it has been demonstrated that pericytes and astrocytes serve important roles in the protection of nervous tissue against oxidative stress following ischemia, by facilitation of BBB repair and attenuation of neuroinflammatory response, respectively (38-41). In particular, Honsa et al (20) demonstrated that $\mathrm{HCN}$ channels were markedly expressed by reactive astrocytes in the CA1 subfield of the rat hippocampus following global cerebral ischemia induced by transient bilateral common carotid artery occlusion combined with hypoxia and that, in this model, electrophysiological properties of reactive astrocytes were significantly altered following ischemia; they concluded that $\mathrm{HCN}$ channels may participate 



Figure 5. HCN2 immunohistochemistry in the hippocampal CA1 region. HCN2 immunohistochemistry in the CA1 subfield of the (A) sham and (B-F) ischemia-operated groups. HCN2 immunoreactivity is distinctly increased in CA1 pyramidal neurons $6 \mathrm{~h}$ after tgCI. Thereafter, HCN2 immunoreactivity is decreased with a time-dependent manner and very weakly observed in CA1 pyramidal neurons 4 days after tgCI. Scale bar, $50 \mu \mathrm{m}$. The asterisks and the arrows represent the CA1 pyramidal neurons and non-pyramidal cells, respectively. (G) ROD is presented as percentages of HCN2 immunoreactive structures in the CA1 subfield following tgCI ( $\mathrm{n}=7$ at each time point). ${ }^{*} \mathrm{P}<0.05$ vs. the sham operated group. ${ }^{\mathrm{H}} \mathrm{P}<0.05$ vs. the previous timepoint group. Bars indicate the means \pm standard error of the mean. (H-M) Double immunofluorescence staining for (H and K) HCN1 (green), (I) Kir6.1 (red), (L) GFAP (red) and $(\mathrm{J}$ and $\mathrm{M})$ merged images in the stratum pyramidale 4 days after tgCI. HCN1 immunoreactivity was localized in Kir6.1-immunoreactive pericytes and GFAP-immunoreactive astrocytes. Arrows denote Kir6.1-immunoreactive pericytes and GFAP-immunoreactive astrocytes in the respective images. Scale bar, $20 \mu \mathrm{m}$. HCN2, hyperpolarization-activated cyclic nucleotide-gated 2; CA1, Cornu Ammonis 1; tgCI, transient global cerebral ischemia; ROD, relative optical density; Kir6.1, ATP-sensitive inward rectifier potassium channel 8; GFAP, anti-glial fibrillary acidic protein.

in the regeneration of ischemic tissue and ionic homeostasis maintenance.
There are certain important limitations of the present study. Firstly, data concerning behavioral and cognitive changes 

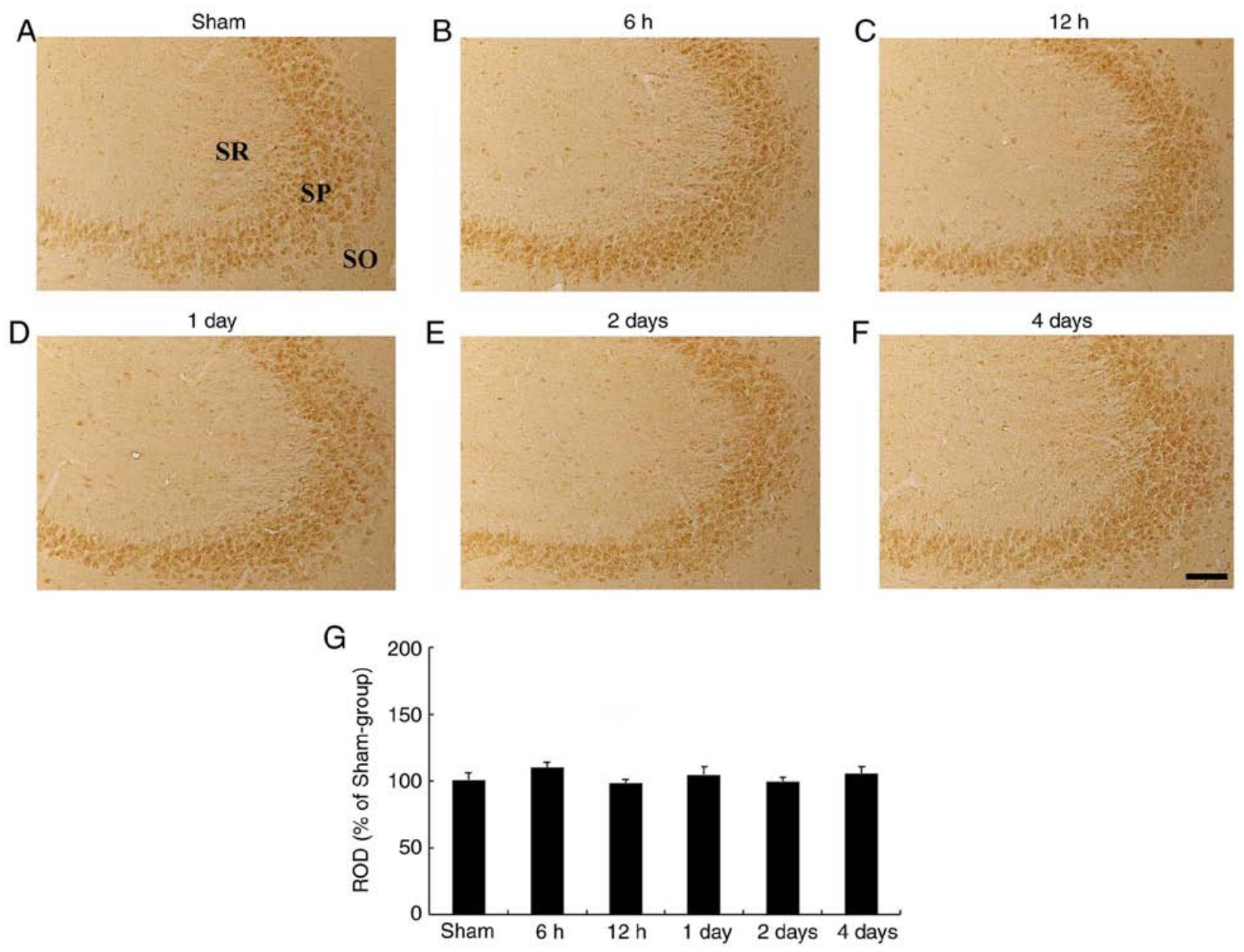

Figure 6. HCN2 immunohistochemistry in the hippocampal CA3 region. (A-F) HCN2 immunohistochemistry in the CA3 subfield of the (A) sham- and (B-F) ischemia-operated groups at (B) $6 \mathrm{~h},(\mathrm{C}) 12 \mathrm{~h}$, (D) 1 day, (E) 2 days and (F) 4 days. HCN2 immunoreactivity levels were not altered in the CA3 subfield following tgCI. Scale bar, $50 \mu \mathrm{m}$. (G) ROD is presented as percentages of $\mathrm{HCN} 2$ immunoreactive structures in the CA3 subfield following tgCI ( $\mathrm{n}=7$ at each time point). HCN2, hyperpolarization-activated cyclic nucleotide-gated 2; CA3, Cornu Ammonis 3; SO, stratum oriens; SP, stratum pyramidale; SR, stratum radiatum; ROD, relative optical density.

following tgCI were not described, although it has been established the tgCI-induced loss of hippocampal CA1 pyramidal neurons leads to impairment of hippocampal-dependent learning and memory and that significant changes in motor behavior are exhibited at 1 day after $\operatorname{tgCI}$ in gerbils $(29,42)$. Secondly, the present study did not investigate how HCN1 and $\mathrm{HCN} 2$ expression affected the death of CA1 pyramidal neurons following $\operatorname{tgCI}$ at a molecular level. Therefore, further studies are required to investigate the functional effects of $\mathrm{HCN} 1$ and/or HCN2 on tgCI-induced neuronal death.

In brief, $\mathrm{HCN} 1$ and $\mathrm{HCN} 2$ immunoreactivities were altered in pyramidal neurons of the hippocampal CA1 subfield in a time-dependent manner following tgCI, and $\mathrm{HCN} 1$ and HCN2 immunoreactivity began to be observed in pericytes and astrocytes in ischemic CA1 subfield at 4 days after tgCI. These results indicate that $\mathrm{tgCI}$-induced changes in $\mathrm{HCN} 1$ and HCN2 expression levels may be closely associated with tgCI-induced DND of pyramidal neurons in the hippocampal CA1 subfield.

\section{Acknowledgements}

Not applicable.

\section{Funding}

The present study was performed with the support of 'Cooperative Research Program for Agriculture Science and Technology Development (project no. PJ01321101)' Rural Development Administration, Republic of Korea, by Basic Science Research Program through the National Research Foundation of Korea (NRF) funded by the Ministry of Science, ICT \& Future Planning (grant no. NRF-2017R1A2B4009079), and by the NRF funded by the Ministry of Education (grant no. NRF-2017R1D1A1B03029311).

\section{Availability of data and materials}

All data generated or analyzed during this study are included in this published article.

\section{Authors' contributions}

JHP, DWK, MHW and CHL were responsible for experimental design, data collection, data analysis, and manuscript writing. CWP, YEP and HAL performed the experiments, TKL and JHA performed data analysis and provided critical comments 
throughout the process of the present study. All authors read and approved the final manuscript.

\section{Ethics approval and consent to participate}

All experimental procedures were approved by the Institutional Animal Care and Use Committee at Kangwon National University (approval no. KW-180124-1).

\section{Patient consent for publication}

Not applicable.

\section{Competing interests}

The authors declare that they have no competing interests.

\section{References}

1. Kirino T: Delayed neuronal death in the gerbil hippocampus following ischemia. Brain Res 239: 57-69, 1982.

2. Park JH, Kim YH, Ahn JH, Choi SY, Hong S, Kim SK, Kang IJ, Kim YM, Lee TK, Won MH and Lee CH: Atomoxetine protects against NMDA receptor-mediated hippocampal neuronal death following transient global cerebral ischemia. Curr Neurovasc Res 14: 158-168, 2017.

3. Saito K, Suyama K, Nishida K, Sei Y and Basile AS: Early increases in TNF-alpha, IL-6 and IL-1 beta levels following transient cerebral ischemia in gerbil brain. Neurosci Lett 206 : 149-152, 1996.

4. Sharma SS and Gupta S: Neuroprotective effect of MnTMPyP, a superoxide dismutase/catalase mimetic in global cerebral ischemia is mediated through reduction of oxidative stress and DNA fragmentation. Eur J Pharmacol 561: 72-79, 2007.

5. Sugawara T, Lewén A, Noshita N, Gasche Y and Chan PH: Effects of global ischemia duration on neuronal, astroglial, oligodendroglial, and microglial reactions in the vulnerable hippocampal CA1 subregion in rats. J Neurotrauma 19: 85-98, 2002.

6. Swanson RA, Ying W and Kauppinen TM: Astrocyte influences on ischemic neuronal death. Curr Mol Med 4: 193-205, 2004.

7. Biel M, Wahl-Schott C, Michalakis S and Zong X: Hyperpolarization-activated cation channels: From genes to function. Physiol Rev 89: 847-885, 2009.

8. He W, Xu X, Lv Q and Guo L: Low dose ZD7288 attenuates the ischemia/reperfusion-induced impairment of long-term potentiation induction at hippocampal Schaffer collateral-CA1 synapses. Cell Mol Neurobiol 34: 611-617, 2014.

9. Huang CC and Hsu KS: Reexamination of the role of hyperpolarization-activated cation channels in short- and long-term plasticity at hippocampal mossy fiber synapses. Neuropharmacology 44: 968-981, 2003.

10. Luo P, Lu Y, Li C, Zhou M, Chen C, Lu Q, Xu X, He Z and Guo L: Long-lasting spatial learning and memory impairments caused by chronic cerebral hypoperfusion associate with a dynamic change of $\mathrm{HCN} 1 / \mathrm{HCN} 2$ expression in hippocampal CA1 region. Neurobiol Learn Mem 123: 72-83, 2015.

11. Lupica CR, Bell JA, Hoffman AF and Watson PL: Contribution of the hyperpolarization-activated current $(\mathrm{I}(\mathrm{h}))$ to membrane potential and GABA release in hippocampal interneurons. J Neurophysiol 86: 261-268, 2001.

12. Mellor J, Nicoll RA and Schmitz D: Mediation of hippocampal mossy fiber long-term potentiation by presynaptic Ih channels. Science 295: 143-147, 2002.

13. Nolan MF, Malleret G, Dudman JT, Buhl DL, Santoro B, Gibbs E, Vronskaya S, Buzsáki G, Siegelbaum SA, Kandel ER and Morozov A: A behavioral role for dendritic integration: HCN1 channels constrain spatial memory and plasticity at inputs to distal dendrites of CA1 pyramidal neurons. Cell 119: 719-732, 2004.

14. Monteggia LM, Eisch AJ, Tang MD, Kaczmarek LK and Nestler EJ: Cloning and localization of the hyperpolarization-activated cyclic nucleotide-gated channel family in rat brain. Brain Res Mol Brain Res 81: 129-139, 2000.
15. Robinson RB and Siegelbaum SA: Hyperpolarization-activated cation currents: From molecules to physiological function. Annu Rev Physiol 65: 453-480, 2003.

16. Santoro B, Chen S, Luthi A, Pavlidis P, Shumyatsky GP, Tibbs GR and Siegelbaum SA: Molecular and functional heterogeneity of hyperpolarization-activated pacemaker channels in the mouse CNS. J Neurosci 20: 5264-5275, 2000.

17. Wahl-Schott $\mathrm{C}$ and Biel M: HCN channels: Structure, cellular regulation and physiological function. Cell Mol Life Sci 66: 470-494, 2009.

18. Baruscotti M, Bottelli G, Milanesi R, DiFrancesco JC and DiFrancesco D: HCN-related channelopathies. Pflugers Arch 460: 405-415, 2010.

19. DiFrancesco JC and DiFrancesco D: Dysfunctional HCN ion channels in neurological diseases. Front Cell Neurosci 6: 174, 2015.

20. Honsa P,Pivonkova H,Harantova L, Butenko O,Kriska J,Dzamba D, Rusnakova V, Valihrach L, Kubista M and Anderova M: Increased expression of hyperpolarization-activated cyclic nucleotide-gated $(\mathrm{HCN})$ channels in reactive astrocytes following ischemia. Glia 62: 2004-2021, 2014.

21. Lee CH, Park JH, Cho JH, Ahn JH, Yan BC, Lee JC, Shin MC, Cheon SH, Cho YS, Cho JH, et al: Changes and expressions of Redd1 in neurons and glial cells in the gerbil hippocampus proper following transient global cerebral ischemia. J Neurol Sci 344: 43-50, 2014.

22. Park JH, Shin BN, Ahn JH, Cho JH, Kim IH, Kim DW, Won MH, Hong S, Cho JH and Lee CH: Ischemia-induced changes of PRAS40 and p-PRAS40 immunoreactivities in the gerbil hippocampal CA1 region after transient cerebral ischemia. Cell Mol Neurobiol 36: 821-828, 2016

23. Du X, Wang D, Li Y, Huo X, Li C, Lu J, Wang Y, Guo M and Chen Z: Newly breeding an inbred strain of ischemia-prone Mongolian gerbils and its reproduction and genetic characteristics. Exp Anim 67: 83-90, 2018.

24. Zhu XL, Yan BC, Tang C, Tang C, Qiu GW, Wu Y, Wang Y and Bo P: Neuroprotective effect of Paeoniae Radix Rubra on hippocampal CA1 region of mice induced by transient focal cerebral ischemia via anti-gliosis and anti-oxidant activity. Chin Herb Med 11: 86-91, 2019.

25. Davies LM, MacLellan CL, Corbett DR and Colbourne F: Post-ischemic diazepam does not reduce hippocampal CA1 injury and does not improve hypothermic neuroprotection after forebrain ischemia in gerbils. Brain Res 1013: 223-229, 2004.

26. Shughrue P and Merchenthaler I: Estrogen prevents the loss of CA1 hippocampal neurons in gerbils after ischemic injury. Neuroscience 116: 851-861, 2003.

27. Carpenter JW and Marion CJ: Exotic animal formulary. Elsevier, St. Louis, Missouri, 2018.

28. Ahn JH, Shin BN, Song M, Kim H, Park JH, Lee TK, Park CW, Park YE, Lee JC, Yong JH, et al: Intermittent fasting increases the expressions of SODs and catalase in granule and polymorphic cells and enhances neuroblast dendrite complexity and maturation in the adult gerbil dentate gyrus. Mol Med Rep 19: 1721-1727, 2019.

29. Lee JC, Park JH, Ahn JH, Kim IH, Cho JH, Choi JH, Yoo KY, Lee CH, Hwang IK, Cho JH, et al: New GABAergic neurogenesis in the hippocampal CA1 region of a gerbil model of long-term survival after transient cerebral ischemic injury. Brain Pathol 26: 581-592, 2016.

30. Ahn JH, Song M, Kim H, Lee TK, Park CW, Park YE, Lee JC, Cho JH, Kim YM, Hwang IK, et al: Differential regional infarction, neuronal loss and gliosis in the gerbil cerebral hemisphere following $30 \mathrm{~min}$ of unilateral common carotid artery occlusion. Metab Brain Dis 34: 223-233, 2019.

31. Kirino T, Tamura A and Sano K: A reversible type of neuronal injury following ischemia in the gerbil hippocampus. Stroke 17: 455-459, 1986.

32. Bender RA, Brewster A, Santoro B, Ludwig A, Hofmann F, Biel $M$ and Baram TZ: Differential and age-dependent expression of hyperpolarization-activated, cyclic nucleotide-gated cation channel isoforms 1-4 suggests evolving roles in the developing rat hippocampus. Neuroscience 106: 689-698, 2001.

33. Seo H, Seol MJ and Lee K: Differential expression of hyperpolarization-activated cyclic nucleotide-gated channel subunits during hippocampal development in the mouse. Mol Brain 8: 13, 2015.

34. Notomi $\mathrm{T}$ and Shigemoto R: Immunohistochemical localization of Ih channel subunits, HCN1-4, in the rat brain. J Comp Neurol 471: 241-276, 2004. 
35. Li S, He Z, Guo L, Huang L, Wang J and He W: Behavioral alterations associated with a down regulation of HCN1 mRNA in hippocampal cornus ammon 1 region and neocortex after chronic incomplete global cerebral ischemia in rats. Neuroscience 165: 654-661, 2010.

36. Oh YJ, Na J, Jeong JH, Park DK, Park KH, Ko JS and Kim DS Alterations in hyperpolarization-activated cyclic nucleotidegated cation channel $(\mathrm{HCN})$ expression in the hippocampus following pilocarpine-induced status epilepticus. BMB Rep 45: 635-640, 2012.

37. Brewster A, Bender RA, Chen Y, Dube C, Eghbal-Ahmadi M and Baram TZ: Developmental febrile seizures modulate hippocampal gene expression of hyperpolarization-activated channels in an isoform- and cell-specific manner. J Neurosci 22: 4591-4599, 2002.

38. Cai W, Liu H, Zhao J, Chen LY, Chen J, Lu Z and Hu X: Pericytes in brain injury and repair after ischemic stroke. Transl Stroke Res 8: 107-121, 2017.

39. Sidoryk-Wegrzynowicz M, Wegrzynowicz M, Lee E, Bowman $\mathrm{AB}$ and Aschner M: Role of astrocytes in brain function and disease. Toxicol Pathol 39: 115-123, 2011.
40. Tian W, Sawyer A, Kocaoglu FB and Kyriakides TR: Astrocyte-derived thrombospondin-2 is critical for the repair of the blood-brain barrier. Am J Pathol 179: 860-868, 2011.

41. Yang S, Jin H, Zhu Y, Wan Y, Opoku EN, Zhu L and Hu B Diverse functions and mechanisms of pericytes in ischemic stroke. Curr Neuropharmacol 15: 892-905, 2017.

42. Janać B, Radenović L, Selaković V and Prolić Z: Time course of motor behavior changes in Mongolian gerbils submitted to different durations of cerebral ischemia. Behav Brain Res 175: 362-373, 2006.

This work is licensed under a Creative Commons Attribution-NonCommercial-NoDerivatives 4.0 International (CC BY-NC-ND 4.0) License. 